\title{
COMPARACIÓN DE TÉCNICAS DE ANÁLISIS MULTIVARIADO MEDIANTE SIMULACIÓN PARA CARACTERIZACIÓN DE RECURSOS FITOGENÉTICOS EN FUNCIÓN DE CARACTERES SUSCEPTIBLES A INTERACCIÓN GENOTIPO-AMBIENTE
}

\author{
Zuliani, P. ${ }^{1}$; Defacio, R. ${ }^{2}$; Lavalle, A. ${ }^{1}$ \& Bramardi, S. ${ }^{1,3}$
}

\begin{abstract}
RESUMEN
Los Recursos Fitogenéticos se conservan ex situ en bancos de germoplasma. Para que los mismos sean utilizados por los mejoradores deben ser caracterizados y evaluados. En el banco de Germoplasma del INTA Pergamino se realiza la caracterización y evaluación de poblaciones locales de maíz, donde los datos obtenidos pueden presentarse como matrices de tres vías (poblaciones * variable * ambiente). A partir de datos empíricos de tres vías se generaron datos simulados, los cuales se utilizaron para comparar las técnicas de Análisis de Componentes Principales (ACP), Análisis Factorial Múltiple (AFM) y Análisis de Procrustes Generalizado (APG). La correlación entre las matrices de datos se cuantificó con el coeficiente RV de Escoufier. Los resultados muestran una alta concordancia entre las configuraciones encontradas con las tres estrategias de análisis. Se destaca el potencial que tienen AFM y el APG en el estudio de la interacción genotipo-ambiente multivariada. También se pudo comprobar que el coeficiente RV de Escoufier, puede ser usado como una sencilla herramienta para cuantificar dicha interacción.
\end{abstract}

Key words: datos de tres vías, Análisis Factorial Múltiple, Análisis de Procrustes Generalizado, coeficiente $R V$, Zea mays $L$.

1.- Departamento de Estadística. Universidad Nacional del Comahue. (8300) Neuquén.

Email: matiroc@live.com.ar

2.- Estación Experimental Agropecuaria Pergamino, Instituto Nacional de Tecnología Agropecuaria.

3.- Centro de Investigaciones en Toxicología Ambiental y Agrobiotecnología del Comahue (CONICET -

UNCo).

Manuscrito recibido el 5 de octubre de 2017 y aceptado para su publicación el 23 de agosto de 2018.

Zuliani, P.; Defacio, R; Lavalle, A.; Bramardi, S. Comparación de técnicas de análisis multivariado mediante simulación para caracterización de recursos fitogenéticos en función de caracteres susceptibles a interacción genotipo ambiente FAVE - Ciencias Agrarias 17 (1): 75-86.

CC BY-NC-SA 4.0 (c) (1) () () 


\begin{abstract}
Comparison of multivariate analysis techniques by simulation to characterize plant genetic resources in terms of characters wich are susceptible to genotype-environment interaction.
\end{abstract}

Characterization and evaluation of phytogenetic resources, preserved ex situ in germplasm banks, is needed to allow their use by breeders. Data recorded from the characterization of maize landraces conserved at the INTA Pergamino germplasm bank could be shown as three way matrices (landraces * trait * environment). Simulated data was generated through three way empirical data, which was used to compare Principal Components Analysis (PCA), Multiple Factorial Analysis (MFA) and the Generalized Procrustes Analysis (GPA) techniques. Escoufier's RV coefficient quantified the correlation between data matrices. High concordance between configurations obtained with the three analysis strategies was shown. MFA and GPA demonstrated a highlighted potential for the multivariate genotype-environment interaction study. It was also found that Escoufier's RV coefficient may be used as a simple tool to quantify this interaction.

Key words: three-way data, multiple factor analysis, generalized Procrustes analysis, $R V$ coefficient, Zea mays L.

\section{INTRODUCCIÓN}

Los Recursos Fitogenéticos están constituidos por material genético de origen vegetal que contiene valor real o potencial para la alimentación y la agricultura. Estos recursos son esenciales para la producción agrícola sostenible, y componen la base biológica para la alimentación, los medios de vida y el desarrollo económico. Según la Organización de las Naciones Unidas para la Agricultura y la Alimentación (FAO), su conservación y uso eficaz es fundamental para garantizar la seguridad alimentaria y nutricional, cubriendo necesidades a corto y largo plazo (8).

La conservación de recursos fitogenéticos puede realizarse en su hábitat natural (in situ) o fuera del mismo (ex situ). Dentro de la segunda alternativa, la forma más conocida de conservación son los Bancos de Germoplasma cuya finalidad es asegurar la preservación de una amplia diversidad de especies de cultivo, que incluyen sus variedades silvestres afines y especies relacionadas menos utilizadas, en sistemas de conservación accesibles. Por otro lado, los bancos deben asegurar la disponibilidad continua de los recursos fitogenéticos para la investigación, el desarrollo tecnológico, la reproducción y la mejora del suministro de semillas para un sistema agrícola sostenible (8).

Los recursos fitogenéticos pueden tener un aprovechamiento directo, tal cual se encuentran conservados, o indirecto, utilizando uno o muy pocos genes, lo cual se logra a través del mejoramiento genético. Sin embargo, para que el germoplasma pueda ser utilizado en los programas de mejoramien- 
to es necesario que, además de su correcta conservación, sea adecuadamente caracterizado y evaluado. Para ello es preciso implementar y desarrollar análisis estadísticos para la caracterización de las poblaciones y el establecimiento de relaciones entre ellas. A partir de esta información, los recursos fitogenéticos debidamente estudiados y catalogados pueden ser fácilmente consultados por los mejoradores en su búsqueda de germoplasma para los programas de mejoramiento (2).

En el caso del maíz, la variabilidad genética presente dentro y entre poblaciones ha sido reconocida como una de las más abundantes del reino vegetal. Gran parte de esta variabilidad es debida a factores unitarios que han sido identificados a través del tiempo y que controlan características fácilmente visibles, tales como los colores, las formas y estructuras. Esta diversidad juega un papel importante, principalmente dentro de los sectores formal e informal de semillas. Dentro del sector formal, las semillas son recolectadas desde sus centros de diversidad para ser almacenadas en bancos de germoplasma, los cuales son integrados a programas de selección en estaciones experimentales. Con este fin, se seleccionan en masa las plantas que son más resistentes a virosis, condiciones climáticas, plagas y que desarrollen un buen porte, para cruzarse con otras plantas de maíz que aporten características determinadas que se quieran conseguir como mejora (13). Concluido el proceso, se obtienen variedades genéticamente homogéneas, altamente demandantes en insumos, adaptadas a la agricultura extensiva, las cuales de forma general son clasificadas como variedades "mejoradas".

En Argentina, en la década de 1950, el Grupo de Mejoramiento de Maíz de la Estación Experimental Agropecuaria (EEA) del Instituto Nacional de Tecnología Agropecuaria (INTA) Pergamino, comienza con las colectas de poblaciones locales y, en 1969 se crea el Banco Activo de Germoplasma (BAP) de maíz el cual actualmente conserva alrededor de 2500 entradas que representan una amplia variabilidad.

En la evaluación agro-morfológica de poblaciones nativas de maíz disponibles en los Bancos de Germoplasma se registran caracteres, generalmente cuantitativos, que pueden ser influenciados por el ambiente. Por este motivo, es importante utilizar un diseño experimental adecuado que permita considerar este efecto. Además de ello es conveniente evaluar, estimar y separar el efecto del ambiente y sus interacciones para así analizar sólo las diferencias debido al genotipo; donde, una gran interacción genotipo por ambiente reduce el progreso genético durante la selección $(11 ; 14)$.

En los ensayos de caracterización de maíz realizados por el banco de germoplasma de INTA, se selecciona un subconjunto del total de poblaciones conservadas el cual se evalúa en dos ambientes, recolectándose información de un cierto número de caracteres. De esta manera y en términos estadísticos, se cuenta con un diseño donde las interrelaciones entre individuos y variables están medidas en dos ambientes, por lo tanto, la información se presenta en dos tablas de individuos por variables, constituyendo lo que se denominan datos de tres vías.

Como alternativas de análisis para este tipo de datos, se proponen comúnmente las siguientes técnicas. En primer lugar, tal como se realiza tradicionalmente, Análisis de Componentes Principales (ACP) sobre los valores medios correspondientes a las variables en ambas tablas, como la forma más simplificada de obtener una estructura media (resumen empírico) de las pobla- 
ciones evaluadas. Por otro lado, es posible aplicar Análisis Factorial Múltiple (AFM) (7), y Análisis de Procrustes Generalizado (APG) $(5 ; 9)$, las dos técnicas más empleadas en el tratamiento de datos de tres vías. Ambas pertenecen a paradigmas de estudio diferentes, el primero es un método de la escuela francesa y el segundo de la escuela anglosajona. El AFM basa sus algoritmos en el álgebra matricial mientras que el APG, es principalmente de carácter geométrico. En ambos casos, es posible obtener una configuración de consenso que establece una estructura media para los dos ambientes en que fueron evaluadas las poblaciones nativas de maíz, y a través de ella es posible caracterizar dichas poblaciones.

En estudios previos $(15 ; 16$; 17) se realizaron diversos análisis con datos empíricos cuya finalidad fue comparar los resultados obtenidos a partir de la aplicación de diferentes técnicas. Por lo tanto, las diferencias, ventajas y similitudes entre los métodos se estudiaron en el contexto de conjuntos de datos reales sin posibilidad de generalización. Para poder generalizar una primera conclusión, referida sólo a la magnitud de las interacciones genotipo-ambiente encontradas en los datos empíricos, en esta investigación se realizaron simulaciones con distintas situaciones del efecto interacción. El estudio de este efecto en el contexto de la caracterización de recursos genéticos es relevante debido a la gran cantidad de diseños de investigación actuales en el que está presente. Los resultados sugieren que se puede utilizar cualquiera de las tres técnicas ya que existe una alta concordancia entre las configuraciones encontradas (configuración consenso en AFM y APG, resumen empírico en ACP); además de mostrar el potencial que tienen AFM y APG en el análisis de la interacción entre genotipo y ambiente.

\section{MATERIALES Y MÉTODOS}

Los datos empíricos utilizados como base para realizar las simulaciones, corresponden a 31 poblaciones locales de maíz (Zea mays L.) de la raza Cristalino Colorado colectadas en la provincia de Buenos Aires, conservadas en el BAP. La evaluación del material se realizó mediante un ensayo a campo siguiendo un diseño con dos repeticiones, y en dos localidades, Pergamino (EEA INTA) y Ferré (Escuela Agrotécnica Salesiana) (4)

Con base en la lista de descriptores para maíz del IBPGRI (International Board for Plant Genetic Resource) (3), se seleccionaron y registraron 10 caracteres que se describen en la Tabla 1.

Es decir, son datos de caracterización de 31 poblaciones nativas de maíz (primera vía) a partir de 10 variables cuantitativas (segunda vía) en dos ambientes diferentes (tercera vía).

La simulación consiste en generar tablas de tres vías, basándose en un modelo de interacción genotipo ambiente del tipo cruzada, estimando los efectos y la variabilidad a partir de los datos empíricos. Sobre las diferentes tablas generadas se aplicaron las técnicas a comparar.

\section{ACP de la tabla promedio}

Dado un conjunto de $n$ individuos y $p$ variables, el Análisis de Componentes Principales (ACP) busca representar adecuadamente esta información en un número menor de variables construidas como combinaciones lineales de las originales, representando óptimamente las observaciones en un espacio de dimensión reducida, lo cual permite identificar variables latentes 
Tabla 1: Caracteres agronómicos-morfológicos.

\begin{tabular}{|lc|}
\hline \multicolumn{1}{|c|}{ Código } & Carácter \\
\hline ALTPL $^{1}$ & Altura de la planta $(\mathrm{cm})$ \\
\hline ALTMZ $^{1}$ & Altura de la mazorca $(\mathrm{cm})$ \\
\hline $\mathrm{LMZ}^{2}$ & Longitud de mazorca $(\mathrm{cm})$ \\
\hline $\mathrm{DMZ}^{2}$ & Diámetro de mazorca $(\mathrm{cm})$ \\
\hline $\mathrm{AGR}^{3}$ & Ancho de grano $(\mathrm{mm})$ \\
\hline $\mathrm{LGR}^{3}$ & Largo de grano $(\mathrm{mm})$ \\
\hline PROL & Prolificidad (espigas/planta) \\
\hline REND & Rendimiento (g) \\
\hline GRXM & Granos por metro lineal \\
\hline PESO & Peso de 1000 granos $(\mathrm{g})$ \\
\hline
\end{tabular}

(1) Valor promedio de la observación de 10 plantas.

(2) Valor promedio de la observación de 10 mazorcas.

(3) Valor promedio de la observación de 10 granos.

(12). Además al transformar las variables originales, en general correlacionadas, en nuevas variables no correlacionadas, se facilita la interpretación de los datos.

En el caso que se presenta, la tabla a la cual se aplica el ACP se construye como promedio de las observaciones en los dos ambientes.

\section{Análisis Factorial Múltiple}

El Análisis Factorial Múltiple (AFM), desarrollado por Escofier y Pagès (1990), en el seno de la Escuela Francesa de Análisis de Datos, es un método adaptado al tratamiento de tablas en las que un mismo conjunto de individuos se describe a través de varios grupos de variables. Los grupos de variables pueden surgir de la utilización conjunta de variables de diferente naturaleza, cuantitativas o cualitativas, o cuando las mismas variables son observadas bajo diferentes condiciones. La única restricción es que las variables que integran un grupo sean de la misma naturaleza. Cuando las variables son cuantitativas, como en este caso, el AFM se basa en la metodología de ACP y consta de dos etapas:1) a cada grupo de variables se asocia una configuración euclídea o nube de individuos denominada nube parcial, que será analizada por separado, obteniendo los factores parciales. Es decir, realiza un ACP de cada una de las tablas $\mathrm{X}_{k}$ donde $\mathrm{X}_{k}$ recoge la valoración de las $p$ variables del grupo $k$. 2) se realiza un ACP de la tabla global que resulta de yuxtaponer todas las tablas $\mathrm{X}_{k}$. En este análisis cada tabla es ponderada por el inverso del primer valor propio obtenido en el ACP de dicha tabla. Esta ponderación mantiene la estructura de cada tabla, ya que todas las variables han recibido la misma ponderación, consiguiendo equilibrar la influencia de los grupos, dado que la inercia máxima 
de cada una de las nubes de individuos, definida por los diferentes grupos, vale 1 en cualquier dirección.

El objetivo de esta técnica consiste en poner de manifiesto los principales factores de variabilidad de los individuos, pero en este caso, estando estos últimos descriptos, de manera equilibrada por los distintos grupos de variables.

En este trabajo se cuenta con dos tablas (una por ambiente) de 10 variables agro-morfológicas caracterizando a 31 poblaciones nativas de maíz.

\section{Análisis de Procrustes Generalizado}

El Análisis de Procrustes Generalizado (GPA) propuesto por Gower (1975) armoniza las configuraciones individuales provenientes de cada tabla, a través de pasos algebraicos iterativos que incluyen traslación, rotación y escalado de las coordenadas bajo dos premisas: mantener la distancia relativa entre elementos de las configuraciones individuales y minimizar las sumas de cuadrados entre puntos análogos, es decir, puntos que corresponden al mismo elemento bajo configuraciones diferentes. La configuración de consenso es obtenida como el promedio de todas las configuraciones individuales transformadas.

En términos matriciales, si cada matriz individual $\mathrm{X}_{k}$ origina una configuración $\mathrm{C}_{k}$ $(k=1,2, \ldots, K)$ determinada al aplicar un ACP $\left(\mathrm{C}_{k}\right.$ con $i$ filas y $j$ columnas donde la $i$-ésima fila corresponde a las coordenadas del $i$-ésimo individuo en las $j$ variables), el escalamiento, la rotación y la traslación pueden expresarse matricialmente por la transformación:

$$
\mathrm{Y}_{k} \rightarrow \rho_{k} \mathrm{C}_{k} \mathrm{H}_{k}+\mathrm{T}_{k}
$$

donde $\mathrm{H}_{k}$ es una matriz ortogonal de rotación, ${ }_{k}$ un factor de escala y $\mathrm{T}_{k}$ una matriz de traslación.

Estos tres elementos son calculados minimizando la expresión:

$$
S_{r}=\sum_{i=1}^{31} \sum_{k=1}^{2} \Delta^{2}\left(P_{i}^{k}, G_{i}\right)
$$

donde $\Delta^{2}\left(P_{i}^{k}, G_{i}\right)$ es la distancia Euclídea entre el punto $P_{i}^{k}$ y el centroide de los puntos análogos designado $\mathrm{G}_{\mathrm{i}}$.

Después de la estandarización inicial o la traslación, en cuanto todas las configuraciones han sido transformadas, una iteración es completada. Luego se calcula una configuración de consenso como la media de todas las configuraciones individuales transformadas y una nueva iteración es inicializada. El proceso es repetido hasta que el cambio entre dos pasos consecutivos en las sumas de cuadrados residual ( $\mathrm{Sr}$ ) es menor a un valor en particular. Una tolerancia de convergencia de 0,0001 es considerada satisfactoria (1).

Gower (1975) propone al finalizar el APG realizar un Análisis de la Varianza que permita identificar la participación relativa de los individuos y las condiciones sobre Sr. Es decir, una vez que el proceso iterativo del método culmina, el APG puede ser expresado en forma de tabla de análisis de la varianza.

En esta presentación se aplica APG a la misma estructura de datos a las que se aplicó AFM.

\section{Coeficiente RV de Escoufier}

Teniendo en cuenta las posibles métricas empleadas para medir la distancia entre puntos, el coeficiente RV (6) se puede utilizar con el objetivo de comparar dos matrices de datos. 
Para dos matrices rectangulares $\left(\mathrm{X}_{1}, \mathrm{X}_{2}\right)$ el coeficiente RV es igual a:

$$
\mathrm{RV}\left(\mathrm{X}_{1}, \mathrm{X}_{2}\right)=\frac{\operatorname{traza}\left(\mathrm{X}_{1} \mathrm{X}_{1}^{\prime} \mathrm{X}_{2} \mathrm{X}_{2}^{\prime}\right)}{\sqrt{\operatorname{traza}\left(\left(\mathrm{X}_{1} \mathrm{X}_{1}^{\prime}\right)^{2} \operatorname{traza}\left(\left(\mathrm{X}_{2} \mathrm{X}_{2}^{\prime}\right)^{2}\right)\right)}}
$$

Desde el punto de vista del álgebra lineal, el numerador del coeficiente corresponde al producto escalar entre dos matrices semidefinidas positivas, proporcionando para ese conjunto de matrices la estructura de un espacio vectorial. En ese sentido, el denominador del coeficiente RV es llamado el producto escalar matricial Hilbert-Schmidt, o Schur o Frobenius (10), y el coeficiente $\mathrm{RV}$ es el coseno entre las matrices.

Asume valores entre cero (no hay similaridad entre las dos configuraciones) y uno (las dos configuraciones caracterizan a los individuos de la misma forma), es decir, informa sobre el grado de homotecia que existe entre las nubes de individuos en ambas configuraciones.

\section{Simulación}

Para la simulación se siguieron básicamente los siguientes pasos:

1) Desarrollo del Modelo: Para la generación de los datos simulados, a partir de información empírica se aplicó un modelo lineal, correspondiente a un diseño completamente aleatorizado con dos factores de tratamiento con interacción, y con dos repeticiones, cuya expresión univariada es:

$$
y_{k l i}=\mu+\alpha_{k}+P_{i}+\propto_{k}-P_{i}+\varepsilon_{k l i}
$$

donde: $y_{k l i}$ es cada una de las variables evaluadas para el ambiente $k$, $\mu$ es la media general,
$P_{i}$ es el efecto de la i-ésima población con $\mathrm{i}=1,2, \ldots, 31$,

$\alpha_{k}$ es el efecto del k-ésimo ambiente con $\mathrm{k}=1,2$,

$\propto_{k}-P_{i}$ es el efecto de la interacción genotipo-ambiente,

$\varepsilon_{k l i}$ es el término del error aleatorio

con errores independientes e idénticamente distribuidos normales con media cero y varianzas homogéneas.

A partir de los datos empíricos se comprobaron los supuestos del modelo, y se analizaron las significancias de los efectos población, ambiente e interacción genotipo-ambiente multivariada. Luego los efectos que resultaron significativos fueron estimados como así también se estimó el cuadrado medio del error para cada variable.

2) Simulación: Se realizó a partir de las estimaciones para cada variable de la media general, efecto población y error experimental, y la relación entre las variables genuina a través de la matriz de correlación. Se propusieron distintas situaciones de magnitud del efecto interacción genotipo-ambiente y el efecto ambiente se dejó fijo y genuino (igual al estimado a partir de los datos). En relación a la magnitud de la interacción se tomaron como referencia los valores que se encontraron en el análisis empírico (nivel “medio”), luego esa magnitud se duplicó (nivel "alto”) y se redujo a la mitad (nivel "bajo”). Se realizaron 100 simulaciones para cada una de estas situaciones en lenguaje $\mathrm{R}$, obteniendo en cada simulación dos tablas de individuos por variables $\mathrm{X}_{1} \mathrm{y} \mathrm{X}_{2}$.

3) Experimentación: Obtenidos los datos de tres vías simulados se procedió a aplicar sobre ellos ACP (sobre los valores medios correspondientes a las 10 variables en las 
dos tablas), AFM y APG obteniendo tres configuraciones (resumen empírico en ACP, configuración consenso en AFM y APG)

4) Análisis de resultados: Para comparar las tres configuraciones halladas en el plano principal, se utilizó la correlación entre matrices de distancia euclídea entre los individuos, acompañada de la prueba de Mantel para verificar su significancia. Para cada par de tablas $\mathrm{X}_{1}$ y $\mathrm{X}_{2}$ generadas se obtuvo la matriz de coeficientes RV, junto con el análisis de la varianza para testear su significación. El RV como una herramienta más, permitió medir el grado de similitud entre ellas.

\section{RESULTADOS}

La Tabla 2 muestra las correlaciones promedio entre las matrices de distancia de las configuraciones obtenidas por las tres estrategias de análisis consideradas en cada una de las 100 simulaciones de datos para las situaciones de interacción genotipo-ambiente alta, media y baja, correspondientes a los dos primeros ejes factoriales. Estas correlaciones resultaron ser altamente significativas por el test de Mantel. Entonces, globalmente los resultados indicaron, tal como ocurrió con el análisis empírico, que las configuraciones encontradas para cada una de las tres técnicas, tienen una alta concordancia para los distintos niveles del efecto interacción multivariada.
Se destaca la alta uniformidad de las configuraciones encontradas con AFM y APG, que se traduce en altas correlaciones (próximas a 1) entre ellas. Además, para la magnitud de interacción alta, las correlaciones entre estas dos técnicas fueron superiores a las encontradas entre ACP y cada una de ellas.

También resulta interesante ver que las correlaciones entre las configuraciones obtenidas con AFM y ACP resultaron mayores que entre las encontradas con APG y ACP. Nótese, al respecto que en este caso particular, como las variables son cuantitativas, la metodología del AFM se basa fundamentalmente en el ACP.

La Tabla 3 muestra el valor del coeficiente RV medio entre cada par de tablas, en cada nivel de interacción genotipo-ambiente. Los resultados del ANOVA mostraron que estos valores fueron altamente significativos para los distintos grados de interacción. También en la Tabla 3 se presentan adicionalmente el mínimo y máximo obtenidos. Se observó que para el nivel de interacción "media” (magnitud de interacción correspondiente a los datos empíricos) se sitúa la relación entre los dos ambientes en un nivel intermedio (similar al obtenido con los datos empíricos), poniendo de manifiesto que los dos ambientes presentaron una estructura con similitudes pero también con algunas diferencias. Para el nivel de interacción "baja” se observó que el valor del coeficiente es más alto, y para interacción "alta”

Tabla 2: Correlaciones media sobre las 100 simulaciones para cada nivel de interacción genotipo-ambiente multivariada

\begin{tabular}{|c|c|c|c|}
\hline & Baja & Media & Alta \\
\hline AFM,APG & 0,9753 & 0,9630 & 0,9445 \\
\hline AFM,ACP & 0,9759 & 0,9107 & 0,8127 \\
\hline APG,ACP & 0,9666 & 0,9079 & 0,7808 \\
\hline
\end{tabular}


el valor del coeficiente resultó muy por debajo del valor medio, lo que implica que en la primera situación, tal como era de esperar, hubo mayor similaridad entre los ambientes.

En las Figuras 1, 2, 3 y 4 se presenta a fines ilustrativos los resultados de estas técnicas en el plano principal de los datos empíricos.

\section{DISCUSIÓN Y CONCLUSIONES}

En este artículo se compararon los resultados obtenidos con las técnicas ACP, AFM y APG mediante simulación a partir de datos de tres vías. Se examina el efecto de la interacción genotipo por ambiente considerando tres niveles diferentes (baja, media, alta).

Tabla 3: Coeficiente RV medio sobre las 100 simulaciones para cada nivel de interacción genotipo-ambiente multivariada

\begin{tabular}{|c|c|c|c|}
\hline & Baja & Media & Alta \\
\hline promedio & 0,7673 & 0,5506 & 0,2727 \\
\hline mínimo & 0,6797 & 0,4422 & 0,2019 \\
\hline máximo & 0,8449 & 0,6495 & 0,3442 \\
\hline
\end{tabular}

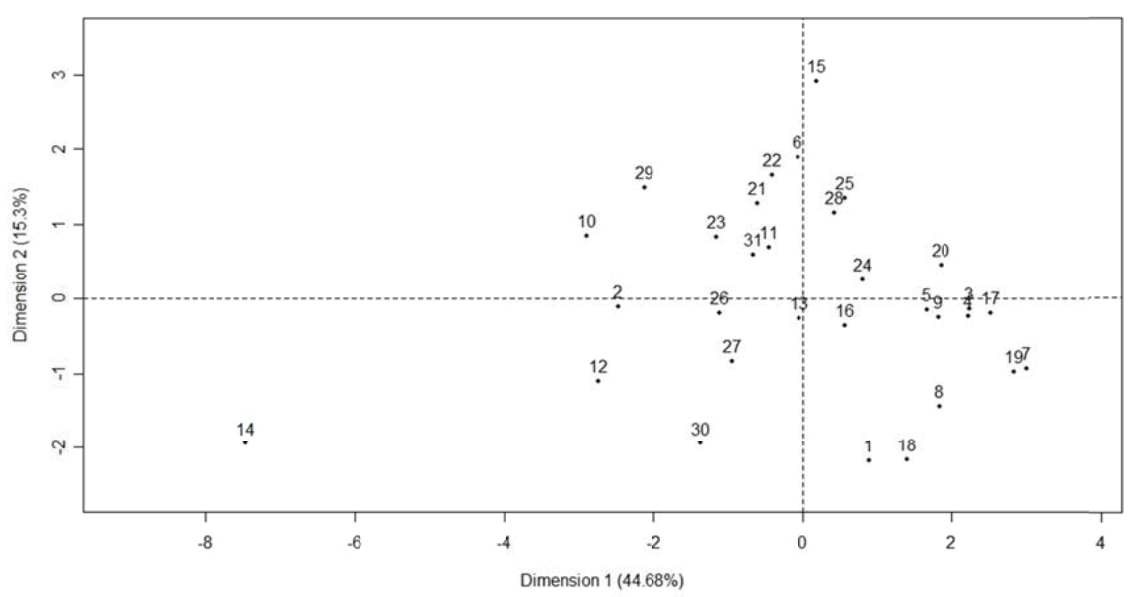

Figura 1. ACP-Proyección de los individuos en el primer plano factorial 
P. Zuliani et al.

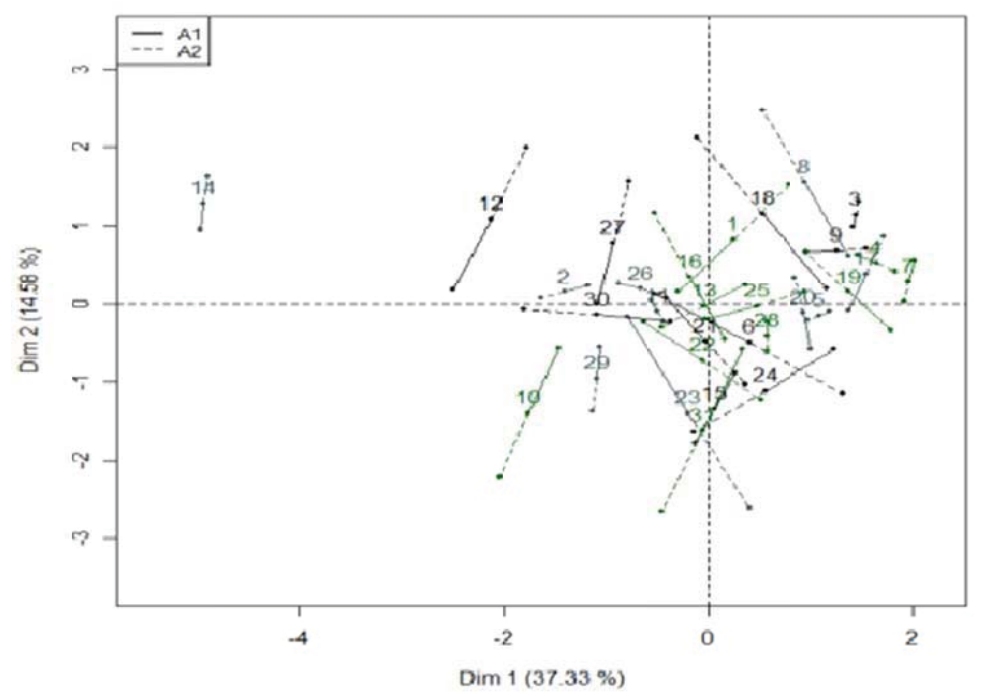

Figura 2. AFM- Visualización de las trayectorias de los individuos

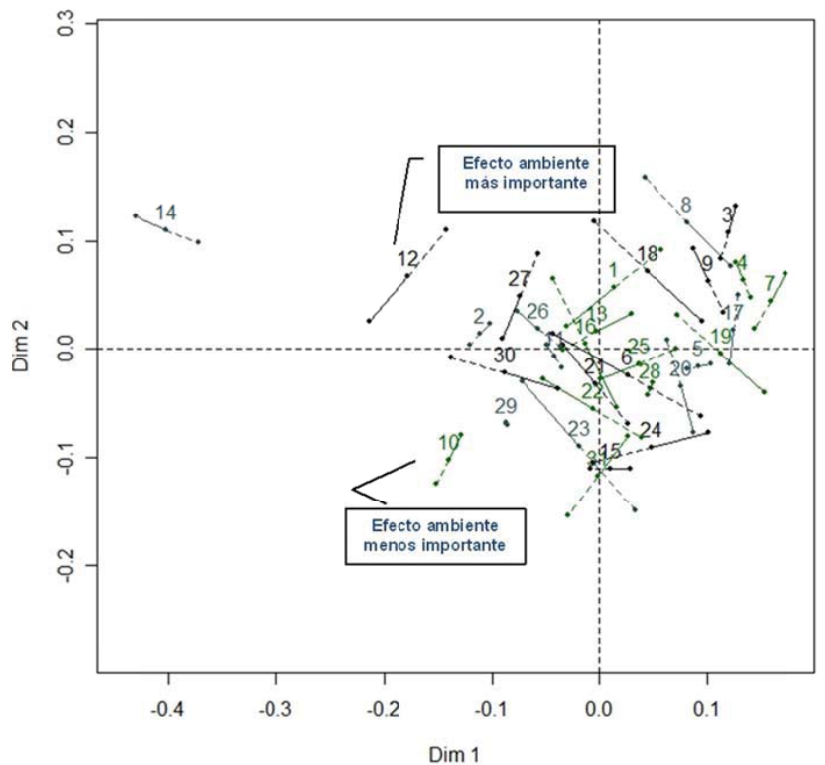

Figura 3. APG-Visualización de las trayectorias de los individuos. 

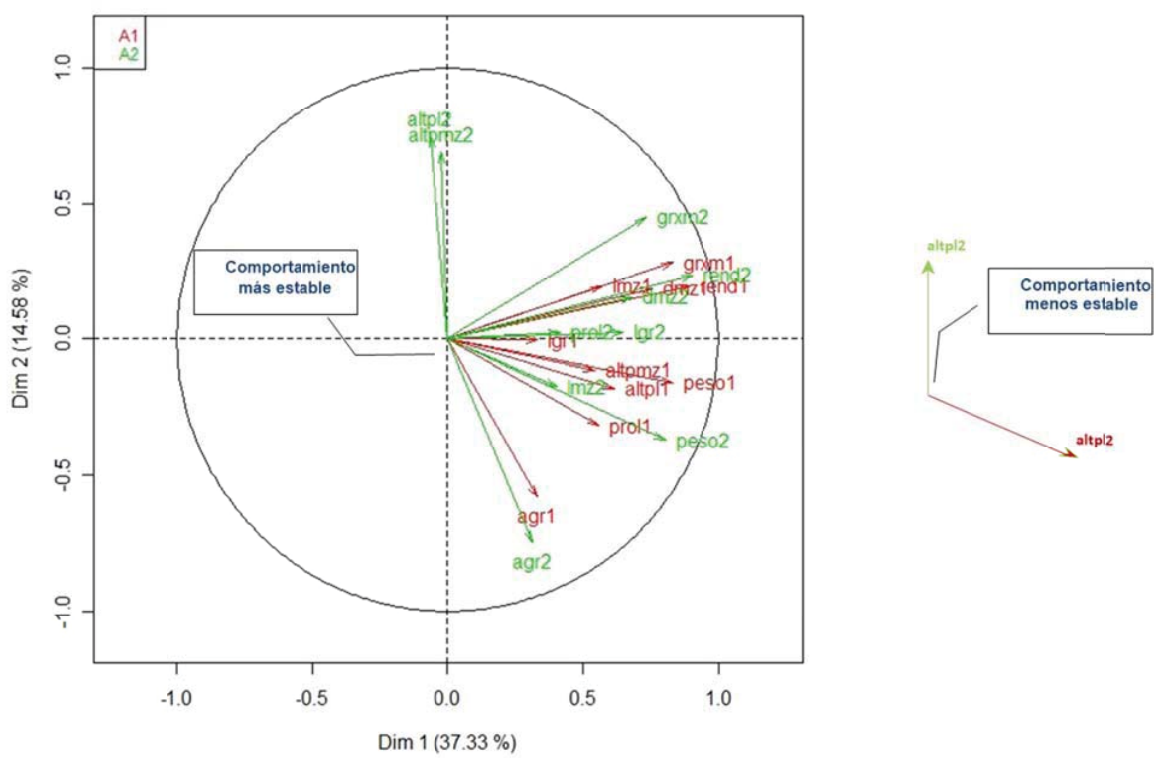

Figura 4. AFM-Proyección de las variables.

Las simulaciones realizadas indican que las tres estrategias de análisis tienen resultados con un alto grado de equivalencia en la configuración final. Sin embargo, AFM y APG en sus rutinas a diferencia del ACP, realizan representaciones superpuestas de los individuos vistos a través de cada una de las dos tablas analizadas (trayectorias de los individuos), las que a partir de sus interpretaciones permiten explicar la interacción genotipo por ambiente a nivel individuo. Es decir, la presencia de individuos con un comportamiento homogéneo en los dos ambientes, contrastada con aquellos que presentan un comportamiento menos homogéneo, permiten evidenciar la existencia de esta interacción (Figura 1, 2 y 3).

Por otro lado, es importante señalar que, AFM a diferencia del APG representa la configuración de las variables en el análisis global. Las variables con un comportamiento estable en los dos ambientes, contrastada con aquellas que presentan un comportamiento menos es- table (a través de un ángulo mayor entre ellas), facilita evidenciar la existencia de interacción variable*ambiente (Figura 4).

Otro resultado importante de este trabajo es que, el coeficiente RV propuesto por Escoufier que cuantifica la homotesis entre configuraciones, resultó ser una herramienta teórica útil, sensible a la interacción genotipo-ambiente. De hecho, este coeficiente toma valores cercanos a cero cuando la interacción es significativa para el vector de p-variables evaluado; y toma valores cercanos a uno cuando la interacción no es importante. Así de esta investigación se pretende aportar una nueva alternativa para el análisis multivariado de la interacción genotipo por ambiente en procesos de caracterización de recursos genéticos. Queda pendiente seguir analizando el comportamiento de estas técnicas ante situaciones donde además de modificar la magnitud general de las interacciones, se consideren distintos patrones de interacción genotipo-ambiente. 


\section{BIBLIOGRAFÍA}

1.- BRAMARDI, S. J.; BERNET, G.; ASÍNS M. J. Y CARBONELL. E. 2005. Simultaneous Agronomic and Molecular Characterization of Genotypes via the Generalised Procrustes Analysis: An Application to $\mathrm{Cu}-$ cumber. Crop Science. 45: 1603-1609.

2.- BRAMARDI, S. J. 2000. "Estrategias para el análisis de datos en la caracterización de recursos genéticos.” Tesis doctoral, dpto. de estadística e investigación operativa. Universidad Politécnica de Valencia, España.

3.- IBPGRI. 1991. Descriptors for Maize. International Maize and Wheat Improvement Center, México city/ International Board for Plant Genetic Resource. Roma. pp.88.

4.- DEFACIO, R. A. 2009. Caracterización y evolución de la variabilidad genética en poblaciones nativas de maíz (Zea mays) de la provincia de Buenos Aires en base a descriptores morfológicos y agronómicos. Tesis de Maestría en Genética. Facultad de Ciencias Agrarias, Universidad Nacional de Rosario. pp. 93.

5.- DIJKSTERHUIS, G. B. Y GOWER, J.C. 1991. The interpretation of Generalizad Procrustes Análisis and allied Methods. Food, Quality and Preference. 67-87.

6.- ESCOUFIER, Y. 1973. Le traitement des variabes vectorielles. Biometrics, 29: 750-760.

7.- ESCOFIER, B. Y PAGÈS, J. 1990. Análisis Factoriales Simples y Múltiples: Objetivos, Métodos e Interpretación. Universidad del País Vasco. Bilbao.

8.- FAO. 2014. Normas para bancos de germoplasma de recursos fitogenéticos para alimentación y agricultura. Edición Revisada. Roma.

9.- GOWER, J. C. 1975. Generalized Procrustes Analysis. Psychometrika, 40: 33-51.
10.- HORN, R. A. Y JOHNSON, C. R. 1985. Matrix Analysis. Cambridge: Cambridge University Press. pp. 291.

11.- MAGARI, R. Y M. KANG. 1993. Genotype selection via a new yield stability statistic in maize yield trials. Euphytica 70:105-111

12.- PEÑA, D. 2002. Análisis de datos multivariantes. Mc Graw Hill. Madrid. 539 pp.

13.- RICCELLI, M. 2000. Mejoramiento Genético y Biotecnología, Introducción a la genética del maíz. En: El maíz. Fundación Polar, Caracas. p. 77-93.

14.- YANG, R. Y BECKER R. 1991. Genotype-environment interactions in two wheat crosses. Crop Science. 31: 83-87.

15.- ZULIANI, R.; LAVALLE, A.; BRAMARDI, S. Y DEFACIO, R. 2011. RV: Un coeficiente para cuantificar la interacción genotipo*ambiente en el análisis de datos de tres modos. XVI Reunión Científica del Grupo Argentino de Biometría. Salta. Resúmenes en CD. Pág. 69.

16.- ZULIANI, P.; LAVALLE, A.; BRAMARDI, S. Y DEFACIO, R. 2012. 'Caracterización de poblaciones nativas de maíz mediante Análisis de Procrustes Generalizado y Análisis Factorial Múltiple’. Revista: Facultad de Ciencias Agrarias. Universidad Nacional de Cuyo (UNCuyo), 44(1):49-64. URL http://bdigital. uncu.edu.ar/4487. ISSN: 0370-4661 (Impreso), 1853-8665 (en línea), ISI Master Journal List.

17.- ZULIANI, R.; LAVALLE, A.; BRAMARDI, S. Y DEFACIO, R. 2012. Alternativas de análisis para datos a tres modos con variables cuantitativas: ACP vs AFM. X Congreso Latinoamericano de Sociedades de Estadística. Córdoba. Resúmenes, pág.75. ISBN 978-9879363-46-1. 